The child and her father have both a heterozygote mutation factor $\mathrm{V}$ Leiden.

1009

\section{RITUXIMAB IN SYSTEMIC LUPUS ERYTHEMATOSUS IN PEDIATRIC PATIENTS}

\author{
M.M. Rodrigues ${ }^{1}$, P. Oliveira ${ }^{2}$, C. Simão²
}

${ }^{1}$ Department of Child and Family - Pediatric Nephrology Unit, Hospital de Santa Maria - Centro Hospitalar Lisboa Norte, ${ }^{2}$ Department of Child and Family - Pediatric Nephrology Unit, Departamento da Criança e da Família - Serviço de Pediatria, Hospital de Santa Maria - Centro Hospitalar Lisboa Norte, Lisbon, Portugal

Systemic Lupus Erythematosus (SLE) is often more severe in children than in adults and innovative approaches are needed for patients who no respond to conventional treatment. Rituximab (RTX) is a chimeric anti CD-20 monoclonal antibody and it has been shown to be a useful therapy in some pediatric autoimmune diseases in which a key role of B cells has been considered.

We report two patients with severe SLE who were refractory to standard drug therapies and our aim was to evaluate the efficacy and safety with this monoclonal antibody.

A 11-years old girl with SLE (cutaneous, musculoskeletal and renal disease), requiring steroids and cyclophosphamide therapy. At 17 years old, renal function decreased (Glomerulonephritis Class IV-Jansen 2004) and refractory pancytopenia leading to more aggressive therapy with steroids, intravenous Immuneglobulin and mycophenolate, without clinical or laboratory response. We decided to add rituximab, achieving satisfactory control of disease, without major side effects.

A 13-years-old boy, with hemolitic-uremic syndrome and neurologic disease who was diagnosed SLE and antiphospholipid antibody syndrome. Conventional therapy (steroids, cyclophosphamide, Immunoglobulin, plasmapheresis) was not enough to control the disease and at last rituximab was introduced. A clinical and laboratorial improvement was achieved in 4 weeks after rituximab administration with remission of disease after 27 months.

Our results suggest the security and efficacy of RTX when used in combination with other immunosuppressive therapies. However, further prospective trials in greater series of children are needed to confirm our results using RTX in monotherapy or co-therapy, and to evaluate adverse events.

\section{0}

\section{ANTIMICROBIAL RESISTANCE IN UROPATHOGENS IN CHILDREN FROM BRAZIL}

\author{
R.C.P. Meneses ${ }^{1}$, M.J.G. Mello ${ }^{2,3}$, J.P. Ribeiro \\ Neto $^{1,3}$, J.B. Correia ${ }^{4,5}$ \\ ${ }^{1}$ Pediatric Nephrology, ${ }^{2}$ Healthcare Control \\ Comission, IMIP-Instituto de Medicina
} Integral Professor Fernando Figueira, ${ }^{3}$ Escola Pernambucana de Saúde, ${ }^{4}$ Research Department, IMIP- Instituto de Medicina Integral Professor Fernando FigueiralMIP-Instituto de Medicina Integral Professor Fernando Figueira, ${ }^{5}$ Paediatric Infectology, Universidade de Pernambuco, Recife, Brazil

Background and aims: To prevent urosepsis and reduce risk of renal scarring in children with urinary tract infection (UTI), empirical antibiotic treatment is started soon after the collection of urine specimens for culture and should be based on local epidemiology of uropathogens and their antimicrobial susceptibility. The aim of this study was to describe pathogens and their antimicrobial resistance in urine cultures of children under five, attending the Instituto de Medicina Integral Professor Fernando Figueira (IMIP), a teaching hospital in Recife, Brazil.

Methods: cross sectional study comparing an earlier (January 1999 to August 2001) and a later (January to December 2008) period of time. It was recorded the urine culture positivity rate, pathogen species; antimicrobial resistance (Kirby-Bauer diffusion disks) of isolates in urine cultures of children with suspected UTI.

Results: 601 (15\%) of 4113 urine cultures were positive. The most frequent isolates in the earlier and later periods were, respectively: E.coli $(30 \%$ and $41 \%)$, Klebsiella spp (27\% and $25 \%), P$. aeruginosa (5\% and $9 \%)$, P. mirabilis $(7 \%$ and $7 \%$ ) and Candida spp. (11\% and $4 \%$ ). In 2008 , the resistance of E.coli was (in descending order): cotrimoxazole $(68 \%)$, nalidixic acid $(52 \%)$, cephalotin (30\%), ciprofloxacin (15\%) and nitrofurantoin $(8 \%)$. When all pathogens are considered, the highest antimicrobial coverages would be reached by ciprofloxacin $(88 \%, C \mid 95 \%=83.4-91.6 \%)$ and nitrofurantoin $(75 \%, \mathrm{Cl} 95 \%=68.6-80.5 \%)$. 
Conclusions: Nitrofurantoin and ciprofloxacin remain viable alternatives, respectively for uncomplicated and complicated UTI. Trends of antimicrobial resistance should be monitored at the local level as important changes occur over time.

1011

\section{EMPLOYMENT CHARACTERISTICS OF PEDIATRIC NURSE PRACTITIONER GRADUATES IN THE USA: AN ADDITIONAL WORKFORCE FOR THE HEALTH CARE OF CHILDREN}

\author{
P.J. Allen \\ School of Nursing, Yale University, \\ New Haven, CT, USA
}

Aims: Describe the characteristics of employment, children cared for, frequency of role functions by practice setting (primary care, specialty practice), and interest in doctoral education of recent PNP graduates.

Method: A 92-item multiple choice and short answer survey tool was sent to graduates from a convenience sample of universities.

Results: The surveys were sent to over 280 graduates with a return rate of $53 \%$ (range $28 \%$ $86 \%$ by university). Of those responding $95 \%$ were employed with $80 \%$ employed full-time. Thirty-two percent were employed in primary care settings with an additional $10 \%$ in school-based clinics. Fiftythree percent were employed in specialty practice in either inpatient or outpatient settings. They often cared for children with acute conditions $(60 \%)$ and chronic health conditions (53\%) irrespective of clinical setting.

Role functions varied by practice setting with provision of preventive health interventions, growth and development counseling, lifestyle behavior counseling, and initiation of referrals being performed more frequently $(p<.05)$ by PNPs in primary care or school-based settings. Provision of case management, staff development, policy and program development, writing for publication, participation in research, system change activities, delegation of care and supervision of students was performed more often $(p<.05)$ by PNPs in hospital settings.

Forty-six percent indicated they were interested in doctoral education with $22 \%$ indicating interest in a
$\mathrm{PhD}$ and $78 \%$ indicating interest in the Doctorate of Nursing Practice.

Conclusion: Pediatric Nurse Practitioners are highly educated and skilled additional pediatric health care providers and should be utilized to their fullest potential.

\section{2}

\section{GOOD NURSING TRAITS: A COMPARISON OF CHILDREN'S VIEWS}

\author{
P. Ferri' ${ }^{1}$, V.L. Napoli' ${ }^{1}$, F. Cerullo \\ ${ }^{1}$ University of Modena and Reggio Emilia, ${ }^{2}$ Hospital \\ of Modena, Modena, Italy
}

Background: Excepting Randall's study (2008), literature about children's feedback of nursing is poor and remarkably dated (Fleitas 1997, Bluebond-Langner 1978). Yet, interviewed children gave a very detailed account, which resulted deeply different from their parents' or care assistants' reports (Miller 2000, Scott 2000).Goal of the study: We interviewed hospitalized children asking for the traits of good nursing. This qualitative study has a phenomenological approach.

Methods: A qualitative interview was used to collect data: we interviewed 10 children admitted at Pediatria of the Azienda Ospedaliera-Universitaria Policlinico in Modena (Italy) in early 2009. Children were not selected according to probabilistic methods. Research was authorized by Unit Direction; we ensured anonymity to parents who gave written consent. The interviewer introduced himself to children explaining the aims of the research and asking them if they were in the mood for answering.

Findings/results: Outcomes may be divided into 4 groups:

1. Natural-born qualities"A good nurse should be a special person in order to help children"

2. Learning from aptitude"A good nurse should be cheerful with children who are afraid of cures"

3. Learning from experience"A good nurse should know and then remember: he should be experienced with children"

4. Cognitive and psycho-physical learning"A good nurse should be qualified, I like nurses who explain what they are doing" 